DEPAEPE (Marc), HELLINCKX (Bart), SIMON

(Frank), The Forgotten Contribution of the Teaching

Sisters. A Historiographical Essay on the Educational

Work of Catholic Women Religious in the 19th and 20th centuries

Louvain : Leuven University Press, 2009, 125 p. (Studia Paedagogica, 44)

Rebecca Rogers

\title{
OpenEdition
}

Journals

Édition électronique

URL : https://journals.openedition.org/histoire-education/2207

DOI : 10.4000/histoire-education.2207

ISSN : 2102-5452

Éditeur

ENS Éditions

Édition imprimée

Date de publication : 1 juillet 2010

Pagination : 131-132

ISSN : 0221-6280

Référence électronique

Rebecca Rogers, «DEPAEPE (Marc), HELLINCKX (Bart), SIMON (Frank), The Forgotten Contribution of the Teaching Sisters. A Historiographical Essay on the Educational Work of Catholic Women Religious in the 19th and 20th centuries ", Histoire de l'éducation [En ligne], 127 | 2010, mis en ligne le 10 mars 2011, consulté le 20 mai 2021. URL : http://journals.openedition.org/histoire-education/2207 ; DOI : https:// doi.org/10.4000/histoire-education.2207

Ce document a été généré automatiquement le 20 mai 2021.

(c) Tous droits réservés 


\section{DEPAEPE (Marc), HELLINCKX (Bart), SIMON (Frank), The Forgotten Contribution of the Teaching Sisters. A Historiographical Essay on the Educational Work of Catholic Women Religious in the 19th and 20th centuries} Louvain : Leuven University Press, 2009, 125 p. (Studia Paedagogica, 44)

Rebecca Rogers

\section{RÉFÉRENCE}

DEPAEPE (Marc), HELLINCKX (Bart), SIMON (Frank), The Forgotten Contribution of the Teaching Sisters. A Historiographical Essay on the Educational Work of Catholic Women Religious in the 19th and 20th centuries, Louvain : Leuven University Press, 2009, 125 p.

(Studia Paedagogica, 44)

1 On ne peut que féliciter les auteurs de ce numéro de Studia Paedagogica de leur recensement fort complet des recherches sur le travail des religieuses catholiques enseignantes à l'époque contemporaine. L'article, d'une cinquantaine de pages, est complété par trente pages de notes et par une bibliographie précieuse de presque quarante pages de références en langue anglaise, française, allemande, néerlandaise, portugaise et espagnole. À partir du dépouillement de vingt revues d'histoire de l'éducation pour la période comprise entre 1985 et 2009, les trois auteurs passent en revue l'action des religieuses enseignantes œuvrant sur quatre continents : l'Amérique du Nord, l'Amérique latine, l'Australie et l'Europe. Le choix a été fait de ne pas prendre 
en compte les études concernant l'activité missionnaire ou coloniale des religieuses, ce qui aurait beaucoup augmenté le nombre de références.

2 L'objectif du volume est d'attirer l'attention des historiens de l'éducation sur le rôle des congrégations dans le processus de féminisation de l'enseignement et de susciter des travaux prenant appui sur les études existantes. Les auteurs abordent leur corpus d'articles et de livres publiés - les mémoires ou les thèses non publiés ne sont pas recensés - de manière thématique. Après un chapitre qui retrace brièvement l'apparition des travaux sur les religieuses depuis les années 1970, les auteurs découpent leur inventaire en six rubriques: la contribution des religieuses à la scolarisation; les congrégations et leurs écoles; leur philosophie éducative, son contenu et sa pratique; la vie et les expériences de vie des enseignantes et de leurs élèves; la professionnalisation de l'enseignement et les transformations du statut des enseignants (la question est ici celle du processus de cléricalisation ou de laïcisation du personnel).

Si certains débats historiographiques sont pointés, comme celui qui porte sur le caractère conservateur (ou non) de l'enseignement des religieuses, ceux-ci ne sont pas au cœur de la présentation dans la mesure où la majorité des travaux existants relèvent de la «micro histoire descriptive» (p. 26). Leur recensement est ainsi un appel à davantage de comparaisons entre les congrégations de pays différents ou entre les activités éducatives des religieuses, celles des religieux et celles des laïques. Les sujets qui restent encore largement à explorer sont signalés, comme le rôle des religieuses dans l'enseignement normal, la pédagogie congréganiste ou l'enseignante congréganiste ordinaire. De même, les approches prometteuses font l'objet de développements, comme l'utilisation de sources non écrites : témoignages oraux ou sources relevant de la culture matérielle (l'architecture et l'imagerie scolaires). Le Canada a une place de choix dans cet inventaire, car les recherches canadiennes sont nombreuses, alors que l'Italie ne fait pas partie du corpus, pour des raisons qui ne sont pas explicitées. Quoi qu'il en soit, le numéro constitue indéniablement un outil de recherche précieux en histoire de l'éducation et un appel à ne pas oublier les «bonnes sœurs » qui ont tant marqué l'enseignement dans de fort nombreux pays. 